\title{
Le savoir du patient en matière de santé le rend plus fort
}

\author{
Hans Kurt \\ Dr, délégué de la FMH et vice-président de la Journée des malades
}

Aujourd'hui, il est difficile de se plaindre d'un manque d'informations médicales. Les questions et les informations concernant notre santé sont omniprésentes: sites internet, blogs, brochures d'information, revues, télévision, YouTube, forums et autres plateformes de conseils. En revanche, il devient de plus en plus difficile de distinguer les informations crédibles et fondées de la publicité cachée, de filtrer les bonnes informations parmi cette masse de données afin d'accroître véritablement notre savoir et ne pas être victimes de désinformation ou de désorientation.

\section{Savoir d'expert et expériences pratiques}

Nos patients ont besoin d'informations fondées, qui leur sont transmises de manière compétente et respectueuse et leur permettent de renforcer leur savoir en matière de maladie et de processus de guérison. Il est avéré que cela contribue à une meilleure santé. Malgré la multitude de sources d'informations, la majorité des patients s'adressent en premier lieu à leur médecin traitant pour lui demander conseil, même s'ils suivent parallèlement d'autres canaux pour s'informer. Pour nous médecins, c'est souvent un véritable défi d'accompagner les patients dans leur quête d'informations et de savoir; en effet, nous ne sommes plus les conseillers, mais uniquement l'une des sources d'informations qu'utilisent les patients pour accéder au savoir. Le médecin n'est plus, non plus, le partenaire omniscient, mais il doit de plus en plus s'efforcer d'apprendre du savoir de son patient. Les patients et leurs proches disposent souvent d'un large savoir, d'expériences personnelles et d'idées qui nous font défaut, à nous les experts médicaux. Cet échange entre savoir d'expert et expériences pratiques permet une mise à niveau des connaissances et sert finalement à la prise en charge personnelle de la maladie et du traitement.

\section{De patient à patient}

Plusieurs études montrent que les patients n'assimilent que 40 à $45 \%$ des informations qui leur sont fournies lors d'entretiens avec des professionnels. D’autres enquêtes consacrées à la charge pesant sur les proches, notamment de personnes atteintes de maladies psychiques, révèlent que $84 \%$ d'entre eux citent comme principale préoccupation les craintes et les soucis provenant d'un manque d'information. Par conséquent, l'un des souhaits les plus urgents consiste à se voir proposer davantage d'informations et de dialogue. Ceci est d'autant plus important que plus de 70\% des proches aidants tombent eux-mêmes malades, le plus souvent de dépression ou de troubles psychosomatiques. Les personnes atteintes de maladies chroniques ou d'un handicap et leurs proches acquièrent de nombreuses connaissances et expériences sur l'évolution de leur cas, et deviennent ainsi des experts de la maladie. Nombre d'entre eux tiennent à partager ce savoir. Cette forme de transfert des connaissances est aujourd'hui partie intégrante des nombreuses offres. Elle est appelée Peer-to-Peer, Peer Counselling ou accompagnement à la guérison. Elle se fonde sur l'idée que les patients sont compétents pour s'aider mutuellement. Les pairs sont crédibles car ils ont traversé, ou traversent, la même expérience. On estime qu'aujourd'hui un patient sur cinq cherche un soutien dans les communautés en ligne. Par ailleurs, il est plus aisé de dévoiler ouvertement ses problèmes dans des groupes d'entraide lorsque l'on parle avec des personnes partageant les mêmes difficultés et lorsque l'on observe un exemple positif qui transmet assurance et espoir. 


\section{Le dilemme du médecin «communicateur»}

Les patients souhaitent participer activement à certains aspects de leur maladie et aux décisions concernant leur santé, ce qui peut être un véritable défi compte tenu du flot d'informations actuel. Lorsqu'un patient ou une patiente ne pose plus de questions, nous partons souvent du principe que tout est clair. Or, il est fréquent que les patients n'aient pas le courage de poser certaines questions ou qu'ils aient l'impression que nous, médecins, n’avons pas le temps de les écou-

\section{La mise en place d'un échange de connaissances} d'égal à égal exige de la confiance et du respect.

ter. Pour nous, la question se pose aussi de savoir quand transmettre une information ou un savoir ainsi que la manière de le transmettre dans un langage approprié pour les patients. Pensons par exemple à la notice explicative des médicaments ou aux déclarations de consentement pour une opération qui s'étendent souvent sur plusieurs pages et qui doivent être signées justement au moment où le patient se trouve déjà en situation de stress lié à l'intervention imminente. De quelle manière pouvons-nous informer des personnes âgées souffrant de troubles cognitifs afin qu'elles comprennent leur état, le traitement qui leur serait bénéfique et celui qu'il serait plus sage d'éviter? Comment pouvons-nous accompagner les migrants compte tenu des difficultés linguistiques souvent considérables? Lorsque des différences culturelles s'ajoutent aux problèmes de langue, les risques d'incompréhension et de malentendu sont grands alors que c'est la compréhension qui est recherchée. Quelles sont les possibilités de traduction, que ce soit à l'aide d'interprètes ou de brochures d'informations existantes dans différentes langues? Comment devons-nous informer lorsque tous les examens possibles ont été effectués et que rien n’a été détecté? Peut-on juste dire: «Vous n'avez rien, ce ne sont que les nerfs?» Et enfin: quelle attitude adopter lorsque nous, médecins, ne sommes pas en mesure de répondre à une question, ce qui est assez fréquent malgré les progrès de la médecine?

Le transfert de connaissances est bien souvent difficile; pour nous, médecins, il est parfois aussi désagréable et pénible. Nous connaissons tous la situation dans laquelle nous devenons des messagers du «malheur». In- former un patient d'une suspicion de cancer et impliquer ses proches est une épreuve lourde qui nécessite du temps pour déterminer le moment, la manière et le contenu de la communication afin que cette dernière soit véritablement utile pour le patient et ses proches. Avec des personnes souffrant de troubles psychiques (psychoses ou trouble borderline), de maladies chroniques graves comme le diabète, ou de maladies accompagnées de douleurs, le transfert de connaissances s'avère également délicat, car il ne se limite pas à un seul entretien, mais exige beaucoup de patience. En particulier dans le cas de maladies difficiles, une bonne information et un bon transfert du savoir permettent de garantir l'adhérence au traitement. Et qu'en est-il de l'affront personnel que nous ressentons lorsqu'un patient demande un second avis médical auprès d'un collègue sans oser nous l'avouer? Et comment supportons-nous les patients et les proches qui savent toujours mieux que les autres?

\section{Respect et rencontre d'égal à égal}

$\mathrm{Si}$, comme l'indique le titre, le savoir de nos patients les rend plus fort dans leur santé, nous pouvons ajouter que l'échange de savoir entre le patient et son médecin les renforce tous les deux dans leurs compétences. Le transfert n'est pas à sens unique; le patient lui-même peut nous enrichir de ses propres connaissances. Il ne s'agit plus d'un simple transfert de connaissances, mais d'un échange respectueux de savoir. Cela implique une écoute attentive, du temps et des propositions régulières d'échange. La mise en place d'un tel processus exige de la confiance et du respect. A ces conditions, les médecins ne sont plus les seuls experts des maladies et de la santé; les patients et leurs proches sont également les experts de leur souffrance et de leur processus de guérison.

\footnotetext{
"La Journée des malades» est une association d'intérêt généra fondée en 1939. Elle se compose de différents membres dont la FMH qui assume la vice-présidence au sein du comité de l'association. La Journée des malades a lieu tous les ans début mars (3.3.2019). En 2018, Madame Doris Fischer Deschler a succédé à la présidence à Veio Zanolini de Minusio qui avait assumé cette fonction pendant de longues années. www.journeedesmalades.ch
} 